\title{
Exogenous Cost Allocation in Peer-to-Peer Electricity Markets
}

\author{
Baroche, Thomas; Pinson, Pierre; Le Goff Latimier, Roman; Ben Ahmed, Hamid
}

Published in:

IEEE Transactions on Power Systems

Link to article, DOI:

10.1109/TPWRS.2019.2896654

Publication date:

2019

Document Version

Peer reviewed version

Link back to DTU Orbit

Citation (APA):

Baroche, T., Pinson, P., Le Goff Latimier, R., \& Ben Ahmed, H. (2019). Exogenous Cost Allocation in Peer-toPeer Electricity Markets. IEEE Transactions on Power Systems, 34(4), 2553-2564.

https://doi.org/10.1109/TPWRS.2019.2896654

\section{General rights}

Copyright and moral rights for the publications made accessible in the public portal are retained by the authors and/or other copyright owners and it is a condition of accessing publications that users recognise and abide by the legal requirements associated with these rights.

- Users may download and print one copy of any publication from the public portal for the purpose of private study or research.

- You may not further distribute the material or use it for any profit-making activity or commercial gain

- You may freely distribute the URL identifying the publication in the public portal

If you believe that this document breaches copyright please contact us providing details, and we will remove access to the work immediately and investigate your claim 


\title{
Exogenous Cost Allocation in Peer-to-Peer Electricity Markets
}

\author{
T. Baroche, P. Pinson, Senior Member, IEEE, R. Le Goff Latimier, H. Ben Ahmed
}

\begin{abstract}
The deployment of distributed energy resources, combined with a more proactive demand side management, is inducing a new paradigm in power system operation and electricity markets. Within a consumer-centric market framework, peer-to-peer approaches have gained substantial interest. Peerto-peer markets rely on multi-bilateral negotiation among all agents to match supply and demand. These markets can yield a complete mapping of exchanges onto the grid, hence allowing to rethink the sharing of costs related to the use of common infrastructure and services. We propose here to attribute such costs through exogenous network charges in several alternative ways i.e. uniformly, based on the electrical distance between agents and by zones. This variety covers the main grid physical and regulatory configurations. Since attribution mechanisms are defined in an exogenous manner to affect each P2P trade, they eventually shift the market issue to cover the grid exploitation costs. It can even be used to release the stress on the grid when necessary. The interest of our approach is illustrated on a test case using the IEEE 39 bus test system, underlying the impact of attribution mechanisms on trades and grid usage.
\end{abstract}

Index Terms-Economic dispatch, Distributed optimization, Optimal power flow, Cost allocation.

\section{NOMENCLATURE}

$\Lambda \quad$ Matrix of trade prices $\lambda_{n m}$ of agent $n$ with agent $m$

. $T$ Matrix transpose operator

$\gamma_{n m} \quad$ Network charges of agent $n$ 's trade with agent $m$

$\mathcal{L} \quad$ Set of lines in the power system

$\mathcal{N} \quad$ Set of nodes in the power system

$|\cdot| \quad$ Absolute value operator

$\Omega \quad$ Peer-to-peer market community of $N_{\Omega}$ agents

$\Omega_{c} \quad$ Set of consumers (subset of $\Omega$ )

$\Omega_{g} \quad$ Set of generators (subset of $\Omega$ )

$\Omega_{p} \quad$ Set of prosumers (subset of $\Omega$ )

$\omega_{n} \quad$ Set of partners of agent $n$

$\mathbf{P} \quad$ Matrix of trade powers $p_{n m}$ of agent $n$ with agent $m$

$\theta_{i} \quad$ Voltage angle at node $i$

$\underline{p_{n}} / \overline{p_{n}}$ Lower/upper power boundary of agent $n$

$\overline{d_{n m}} \quad$ Electrical distance between agent $n$ and agent $m$

$f_{n} \quad$ Cost function of agent $n$

$N_{n m}^{\text {zone }} \quad$ Number of zones between agent $n$ and agent $m$

$p_{n} \quad$ Total amount of power traded by agent $n$

$u \quad$ Network fee of cost allocation policy

T. Baroche (corresponding author) and R. Le Goff Latimier , and H. Ben Ahmed are with the SATIE Laboratory located at Ecole Normale Superieure of Rennes, France, e-mail: thomas.baroche@ens-rennes.fr, roman.legofflatimier@ens-rennes.fr, hamid.benahmed@ens-rennes.fr.

P. Pinson is with the Center for Electric Power and Energy, Danmarks Tekniske Universitet, Denmark, e-mail: ppin@dtu.dk.

The authors are partly supported by the Danish Innovation Fund and the ForskEL programme through the projects ' $5 \mathrm{~s}$ ' - Future Electricity Markets (12-132636/DSF), CITIES (DSF-1305-00027B) and The Energy Collective (grant no. 2016-1-12530)
$Z_{i l} / Y_{i l}$ Element of bus impedance/admittance matrix from node $i$ to node $l$

\section{INTRODUCTION}

Distributed energy resources, jointly with ICT and energy system management for residential homes and buildings, are making us rethink our approach to power system operation. Especially, going down to lower levels of the network, new type of agents are appearing, namely prosumers, with the ability to produce and consume (and most likely store in a very near future). While substantial efforts are made to have power system operation evolve in view of that new context, electricity markets have not gone yet through the same process of accommodating this new context with its challenges and opportunities. Electricity markets are expected to go from producer-centric to consumer-centric [1], [2], while they will most likely include a peer-to-peer (P2P) and communitybased component [3]. A P2P market relies on multi-bilateral direct trades among participants. Employing a P2P market framework could yield a number of advantages, for instance thanks to product preference and its consumer-centric nature, allowing for a wealth of new business models.

Product preference is to be understood here as the fact that market players can express preferences on the type and quality of the energy they will exchange. Such preferences could be for local energy generation, for energy with limited $\mathrm{CO}_{2}$ emissions, etc. However, there may be discrepancies between market-clearing (and related dispatch) and feasible dispatch in view of grid-related and operational constraints. In parallel, while it appears normal to socialize grid-related costs in the current wholesale-retail market structure, a future with peerto-peer exchange and preferences may allow to rethink the way we attribute such costs. Our objective here is hence to describe a consumer-centric market allowing to allocate gridrelated costs in an exogenous manner. Grid related costs may refer to network investment cost as well as operating costs such as maintenance, power losses, etc.

The various attribution mechanisms are to impact trades and subsequent network usage. The first approach to coordinated multi-lateral electricity trades was already proposed nearly 20 years ago [4]. The original aim was to allow for the separation of economics and reliability of system operation, as is the case for current European pool-based electricity markets. The proposal involved an iterative process with all players proposing their trades first, followed by the system operator to decide whether the trades respect operational constraints, or not. This proposal was recently enhanced in [5], also analyzing game-theoretical properties of the solutions obtained. In both 
cases, the authors pointed at the fact that charges for network usage were not considered.

A second approach may consist in relying on optimal power flow (OPF) models, allowing to consider network constraints in an endogenous manner (see e.g. [6]). While those are traditionally solved in a centralized fashion, many decomposition techniques were proposed to solve them in a distributed fashion. Based on approximate Newton directions [7] proposed a decentralized method to solve optimal power flow control for power systems with overlapping areas. [8] followed by [9] respectively proposed distributed state estimation and multiagent coordination in micro-grids based on consensus and innovation approach. Concurrently [10] used the alternating direction method of multipliers (ADMM), developed by [11], to solve optimal power flow in a distributed manner. [12] did the same with another consensus-based mechanism and applied it to energy management of cooperative micro-grids with $\mathrm{P} 2 \mathrm{P}$ energy sharing in [13]. A comparison of different distributed and decentralized algorithms was finally made in [14]. More recently, works like that of [15] proposed to account for network limits in the presence of distributed renewable resources and using decentralized consensus on a blockchain. Even though those operational problems are increasingly considered in decentralized manner, these do not comprise a market construct while they do not account for how grid-related usage costs would be attributed.

In network-constrained economic dispatch problems, e.g. [16], nodal prices classically encompasses both energy generation and congestion-related costs. Hence, grid costs to be recovered are only related to congestion and network usage. In contrast, the bilateral contracts considered here have a component that is based on energy generation costs, supplemented by a network charge component. This network charge component is not only to recover all network costs but also other costs e.g. operational, taxes and policy-related costs. Hence, network constraints are not forced directly but rather accommodated through these network charges. Another important benefit of this approach is that market participants have knowledge of network charges prior to the negotiation process. Contrary to a classical economic dispatch, this transparency on network charges enables agents to anticipate on what it will cost them to trade on the network. The resulting P2P market formulation comprises a simple tool, transparent to market participants, for system and market operators to limit potential detrimental effects that might be induced by $\mathrm{P} 2 \mathrm{P}$ markets on power networks. In this paper, costly incentives will be used to allocate grid costs related to the $\mathrm{P} 2 \mathrm{P}$ market. The strategy to allocate costs, also called cost allocation policy, imposed to the P2P market participants is chosen by the system operator. The proposed cost allocation policies will be pondered by a coefficient, named unit fee. The unit fee will give a degree of freedom for the system operator to reach cost recovery.

The manuscript is structured as follows. Firstly, a novel P2P market formulation allowing for product preference is proposed in Section II. It is eventually solved in a decentralized manner based on consensus ADMM. Subsequently, Section III introduces three designs of network charges based on product preference to influence the P2P market negotiation mechanism.
Simulation results are presented and discussed in Section IV using a test case relying on the IEEE 39-bus system. Section $\mathrm{V}$ gathers our conclusions and perspectives for further work.

\section{P2P MARKet Design AND CORRESPONDING OPTIMIZATION PROBLEM}

A P2P market is based on a community of agents with flexible consumption or production. In this paper agents are supposed rational as in [17], i.e. always objectively taking the most beneficial decisions, and non-strategic, i.e. not anticipating actions and reactions of other agents. The proposed formulation will be compatible with the presence of prosumers inside the community. Emphasis is eventually placed on a deterministic clearing mechanism for a single market time unit. It may readily be extended to multiple time units with temporally binding constraints, while uncertainty could also be considered in a scenario-based stochastic optimization framework. First, a P2P market structure is described and is, then, translated into a decentralized consensus ADMM inspired from [11].

\section{A. Problem Formulation}

This paper aims at proposing an alternative way to treat the following endogenous $\mathrm{P} 2 \mathrm{P}$ economic dispatch

$$
\begin{aligned}
& \min _{\mathbf{P}, p_{n} \in \Omega, \theta_{i \in \mathcal{N}}} \quad \sum_{n \in \Omega} f_{n}\left(p_{n}\right) \\
& \text { s.t. } \quad \mathbf{P}=-\mathbf{P}^{\top} \\
& p_{n}=\sum_{m \in \omega_{n}} p_{n m} \quad n \in \Omega \\
& \underline{p_{n}} \leqslant p_{n} \leqslant \overline{p_{n}} \quad n \in \Omega \\
& p_{n m} \geqslant 0 \quad n \in \Omega_{g} \\
& p_{n m} \leqslant 0 \quad n \in \Omega_{c} \\
& \underline{p_{n}} \leqslant p_{n m} \leqslant \overline{p_{n}} \quad n \in \Omega_{p} \\
& q_{i j}=Y_{i j}\left(\theta_{j}-\theta_{i}\right) \leqslant C_{i j} \quad(i, j) \in \mathcal{L}(1 \mathrm{~h}) \\
& \sum_{n \in \mathcal{N}_{i}} p_{n}=\sum_{(i, j) \in \mathcal{L}} q_{i j} \quad i \in \mathcal{N}
\end{aligned}
$$

which straightly includes transmission network constraints, as in [16], in the context of a P2P market. In transmission networks, the admittance of electrical lines $\mathcal{L}$, noted $Y_{i j}$ for the line connecting node $i$ and $j$, are classically assumed to be driven by their inductance in presence of pure sinusoidal voltage and current. This assumption leads to real power flows $q_{i j}$ proportional to the difference of voltage angles, noted $\theta_{i}$ at node $i$, between the two ends of the line as in (1h). To avoid any damage to transmission lines their flows are bounded by thermal limits $C_{i j}$ related to the heat they can dissipate. Moreover, a power balance must be kept (1i) at each nodes $\mathcal{N}$ of the grid between line flows and power injections of agents connected to it, so in $\mathcal{N}_{i}$ at node $i$.

The goal of the P2P community $\Omega$, of size $N_{\Omega}$, is to minimize the total cost which sums all individual cost functions as in (1a). To minimize its cost function $f_{n}$, agent $n$ is able to optimize its volume traded $p_{n}$ within a flexibility range defined by a lower $p_{n}$ and an upper $\overline{p_{n}}$ bound, as expressed in (1d). Traded amount $p_{n}$ is taken positive if agent $n$ is selling electricity, and negative when buying. Considering 
multi-bilateral trades calls for a split of net powers, in the manner of [18], into a set of multiple bilateral trades $p_{n m}$. Every possible bilateral power trades within the community can be condensed in a matrix $\mathbf{P}$ such that

$$
\mathbf{P}=\left(\begin{array}{ccc}
p_{11} & \cdots & p_{1 N_{\Omega}} \\
\vdots & \ddots & \vdots \\
p_{N_{\Omega} 1} & \cdots & p_{N_{\Omega} N_{\Omega}}
\end{array}\right)
$$

where $p_{n m}$ is necessarily equal to zero if agent $m$ is not in agent $n$ 's trading partnership set $\omega_{n}$. Net powers are then obtained by $p_{n}=\sum_{m \in \omega_{n}} p_{n m}$ as in (1c). As outlined in (1b), $\mathbf{P}$ is skew-symmetric to insure power balance of each trade, so $p_{n n}=0$. This allows to potentially individualize prices per trade.

In this situation agents can buy a large amount of energy at a low price from one agent to sell it back at a higher price to another. This possibility of arbitraging can be proscribed by limiting the possible amounts traded. Generators, for which $\underline{p_{n}} \geqslant 0$ and grouped in $\Omega_{g}$, are forbidden to buy power in $\overline{(1 e)}$. On the other hand consumers, for which $\overline{p_{n}} \leqslant 0$ and grouped in $\Omega_{c}$, are forbidden to sell power in (1f). However, prosumers, gathered in $\Omega_{p}$, must still be able to either buy or sell power since they are such that $p_{n}<0<\overline{p_{n}}$. Power trades of prosumers are bounded by their power boundaries as in $(1 \mathrm{~g})$.

It is essential to notice that contrary to [16] dual variables of nodal power balances (1i), noted $\eta_{i}$, do not include the energy generation price but only prices to derive nodal energy prices. Note that congestion rights originate from (1h)'s dual variable. In our case, energy generation prices are given by the dual variables for trade reciprocity (1b), denoted $\boldsymbol{\Lambda}=\left(\lambda_{n m}\right)$.

Directly coupling the P2P market to grid constraints as in (1) implies an intense involvement of the system operator at each step of the solving algorithm. To level this an exogenous approach of the network limitations could be used. Network constraints (1h)-(1i) can be condensed in a regularization function $h$, equal to 0 if they are respected and $+\infty$ if they are violated. It can be noted that in this case $h$ depends on the real power injections $p_{n \in \Omega}$. Then, problem (1) can be written as

$$
\begin{array}{ccc}
\min _{\mathbf{P}, p_{n \in \Omega}} & \sum_{n \in \Omega} f_{n}\left(p_{n}\right)+h\left(p_{n \in \Omega}\right) & \\
\text { s.t. } & \mathbf{P}=-\mathbf{P}^{\top} & \\
& p_{n}=\sum_{m \in \omega_{n}} p_{n m} & n \in \Omega \\
& \underline{p_{n}} \leqslant p_{n} \leqslant \overline{p_{n}} & n \in \Omega \\
p_{n m} \geqslant 0 & n \in \Omega_{g} \\
p_{n m} \leqslant 0 & n \in \Omega_{c} \\
\underline{p_{n}} \leqslant p_{n m} \leqslant \overline{p_{n}} &
\end{array}
$$

However, even though (3) enables he system operator to recover congestion related costs, it does not guarantee the recovery of the costs of maintenance, modernization of power lines, taxes, and policies. This paper proposes to replace the regularization function $h$ with exogenous terms. These exogenous terms would aim not only at allocating congestionrelated costs but also costs of maintenance and modernization of power lines, taxes, and policies such as e.g. renewable support schemes. Preference prices as introduced in [18] seems a good candidate for this purpose. Then, regularization function $h$ evaluating network constraints is be replaced by a cost allocation function defined as

$$
\Gamma(\mathbf{P})=\sum_{n \in \Omega} \gamma_{n}^{0}+\sum_{m \in \omega_{n}} \gamma_{n m} p_{n m}
$$

where parameter $\gamma_{n m}$ is the network charge associated to power trade $p_{n m}$ for the given time step. Constant terms $\gamma_{n}^{0}$, which do not affect the minimization outcome, allow reflecting costs that are independent of the power traded, such as power line investment and maintenance. Network charges $\gamma_{n m}$, detailed in Section III, would then account for congestion-related costs and taxes. Function $\Gamma$ is separable among participants, and will be integrated in their objective function as it will be further discussed in Section III. Note that $\Gamma$ also represents the amount of money collected by the system operator from community $\Omega$ for its use of the power system.

In problem (3) with (4), reciprocity constraint (3b) is the only barrier to fully distribute it. To overcome this an additional slack variable $\mathbf{W}$ can be considered. Variable $\mathbf{W}$, which can contribute to reach consensus, aims at being the image of all possible trades $\mathbf{P}$. For this, reciprocity constraint (3b) is replaced by power consensus constraint (5b) leading to the deterministic, single time-step, exogenous P2P economic dispatch

$$
\begin{array}{ccc}
\min _{\mathbf{P}, p_{n \in \Omega}, \mathbf{W}} & \sum_{n \in \Omega}\left[f_{n}\left(p_{n}\right)+\gamma_{n}^{0}+\sum_{m \in \omega_{n}} \gamma_{n m} p_{n m}\right] \\
\text { s.t. } & \left(\mathbf{W}-\mathbf{W}^{\top}\right) / 2=\mathbf{P} & n \in \Omega \\
& p_{n}=\sum_{m \in \omega_{n}} p_{n m} & n \in \Omega \\
\underline{p_{n}} \leqslant p_{n} \leqslant \overline{p_{n}} & n \in \Omega_{g} \\
p_{n m} \geqslant 0 & n \in \Omega_{c} \\
p_{n m} \leqslant 0 & n \in \Omega_{p} .
\end{array}
$$

Note that the definition of partnership set $\omega_{n}$ only inform on agent $n$ 's possible trades but does not enforce participation. In other words, agent $n$ keeps the possibility to opt-out of the market, i.e. with outcome $\left(p_{n m}\right)_{m}=0$, if its power boundaries allow it. As proven in Appendix A, when no network charges are applied, i.e. all $\gamma_{n m}=0$, trading prices $\lambda_{n m}$ are uniform and equal to the pool market price. The system operator does not intervene in solving (5) as it only provides network charges $\gamma_{n m}$ a priori. Hence, network charges are provided in a transparent manner before negotiations such that agents can anticipate on the over costs brought by the use of the power system.

\section{B. Decentralized P2P Market}

As developed in Appendix B, a decentralized procedure based on the consensus ADMM of [11] is used to solve (5). This decentralized method solves global problem (5) and, hence, leads to a competitive equilibrium which efficiency strongly depends on the chosen network charges. According 
to [19] ADMM seems well adapted for negotiation mechanisms in smart grids. Several extensions and convergence rate improvements have been proposed in [20]-[23]. Given the focus of this paper is not on scalability a straightforward implementation of consensus ADMM is used.

The final decentralized negotiation mechanism reads

$$
\begin{array}{cc}
P_{n}^{k+1}=\underset{P_{n}}{\operatorname{argmin}} & f_{n}\left(p_{n}\right)+\gamma_{n}^{0}+\sum_{m \in \omega_{n}}\left[\gamma_{n m} p_{n m}\right. \\
+\lambda_{n m}^{k}\left(\frac{p_{n m}^{k}-p_{m n}^{k}}{2}-p_{n m}\right) & \\
\left.+(\rho / 2)\left(\frac{p_{n m}^{k}-p_{m n}^{k}}{2}-p_{n m}\right)^{2}\right] \\
\text { s.t. } \quad p_{n}=\sum_{m \in \omega_{n}} p_{n m} \\
\frac{p_{n} \leqslant p_{n} \leqslant \overline{p_{n}}}{p_{n m} \geqslant 0} & \text { if } n \in \Omega_{g} \\
p_{n m} \leqslant 0 & \text { if } n \in \Omega_{c} \\
\lambda_{n m}^{k+1}=\lambda_{n m}^{k}-\rho\left(p_{n m}^{k+1}+p_{m n}^{k+1}\right) / 2 & \text { if } n \in \Omega_{p}
\end{array}
$$

where penalty factor $\rho>0$. Element $\lambda_{n m}$ of matrix $\boldsymbol{\Lambda}$ corresponds to generation price of electricity for traded volume $p_{n m}$. Possible trades of agents $n$ can be grouped in variable $P_{n}=\left(p_{n m}\right)_{m \in \omega_{n}}$. According to [11], supposing cost functions $f_{n}$ to be closed, proper, and convex is a sufficient condition to ensure convergence of (6). This formulation allows to have primal feasibility of constraints $(5 c)-(5 g)$ at each iteration step. However, primal feasibility of trades reciprocity (5b) is only verified at the limit after convergence. Note that the additional terms of the augmented Lagrangian in (6a) aims at encouraging, economically, an agent $n$ to reach power consensus with its partners. Global stopping criteria associated to (6) are such as

$$
\sum_{n \in \Omega} r_{n}^{k+1} \leqslant \epsilon^{\mathrm{pri}^{2}} \text { and } \sum_{n \in \Omega} s_{n}^{k+1} \leqslant \epsilon^{\text {dual }^{2}}
$$

with, respectively, primal and dual local residuals

$$
\begin{aligned}
r_{n}^{k+1} & =\sum_{m \in \omega_{n}}\left(p_{n m}^{k+1}+p_{m n}^{k+1}\right)^{2} \\
s_{n}^{k+1} & =\sum_{m \in \omega_{n}}\left(p_{n m}^{k+1}-p_{n m}^{k}\right)^{2} .
\end{aligned}
$$

Parameters $\epsilon^{\text {pri }}$ and $\epsilon^{\text {dual }}$ denotes primal and dual global feasibility tolerances, respectively.

The overall negotiation mechanism occurs as follows. Each market participant first solves its own local optimization (6a). Once updated, each agent $n$ individually sends its trade proposals $\left(p_{n m}^{k+1}\right)_{m \in \omega}$ to each partner $m \in \omega_{n}$. After receiving all counter proposals $\left(p_{m n}^{k+1}\right)_{m \in \omega}$, agent $n$ can compute its new trade prices $\left(\lambda_{n m}^{k+1}\right)_{m \in \omega}$ with (6b), and local residuals $\left(r_{n}, s_{n}\right)^{k+1}$ with (8). Finally, each agent $n$ broadcasts its local residuals to all and, when all local residuals $\left(r_{i}, s_{i}\right)_{i \in \Omega \backslash\{n\}}^{k+1}$ are received, tests global stopping criteria (7). This process is repeated until convergence. Being decentralized the negotiation mechanism is not supported by a central entity. However, as most information exchanges, a communication standard needs to be defined by an institutional organization to associate a communication protocol between peers participating in the market.

This type of decentralized negotiation mechanism is believed to require solely local characteristics. However, a deeper analysis based on inverse problem theory [24] should be conducted to verify that exchanges of power proposals does not jeopardize this privacy. As illustrated in [25]-[27], privacy issues go beyond than the sole topic of multi-bilateral trades but also affect smart grids in general. Interestingly, the proposed negotiation mechanism limits the amount of transmitted information as agents only send their trade proposal to their direct partners and their local residuals. In this context it would be interesting to use a secured mechanism, as does [15] for prices updates. In addition, game theory studies on bounded rationality, as in [28], [29], still hold but might need some adaptations.

\section{Exogenous Operation Cost Allocation}

When the goal is to obtain a P2P market with allocation of grid-related costs it is possible to use network charges as in (4). Contrary to preference prices chosen by agents, parameters $\gamma_{n m}$ are provided by the system operator a priori when used as network charges. As mentioned in Section I, remember that, in this paper, a cost allocation policy refers to the way costs are divided between P2P market participants. The proposed cost allocation policies will define how network charges are estimated. They will eventually be pondered by a coefficient named unit fee to allow a level of slackness for the system operator to reach cost recovery. Another objective of network charges may be to reduce congestion risks. In other words, it should allow the system operator to incite agents to behave in a beneficial way for the power system. This property is important because the outcome of (5) does not necessarily satisfy network constraints (1h)-(1i), as shown later in Section IV.

Finally, when an incident occurs on the electric network security dispositions are automated. However, the market as defined initially is not intrinsically considering this deteriorated mode. Partnership sets $\omega_{n}$ could be dynamically adapted in case of congestion. But this would require to duplicate the number of signals sent by the system operator to market participants. In addition, participants would have to manage different routines. A simpler way to influence agents is to apply new unit fees. That way network charges offer an indirect mechanism to handle deteriorated modes. Thus, network charges can push agents to shift from their usual partners to others unaffected by the malfunctions while keeping the same routine. At the limit, this operating mode of cost allocation policies enables market islanding. This corresponds to a security market procedure with the least grid stress while waiting for repairment.

After explicitly expressing the amount of money collected by the system operator, three cost allocation policies are proposed.

\section{A. Total Fees}

The money paid (resp. received) by agent $n$ for buying from (resp. selling to) agent $m$ is given by the perceived price $\mu_{n}=\lambda_{n m}-\gamma_{n m}$, as shown in Appendix A. Network charges represent exogenous costs. Thus, when agent $n$ consumes its $\gamma_{n m}$ are negative which leads to perceived prices $\mu_{n}$ higher 


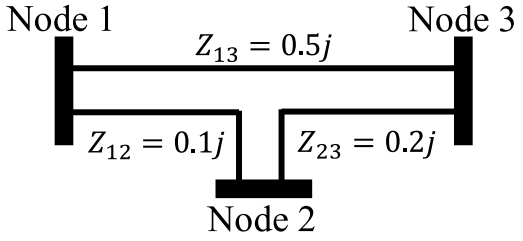

(a) Illustrative test system $\left(j^{2}=-1\right)$

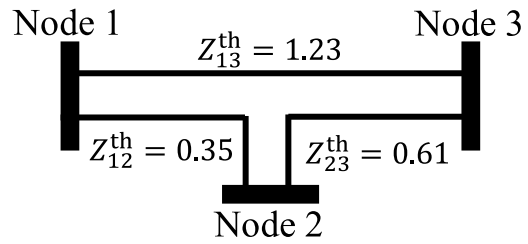

(b) Lines' Thevenin impedance

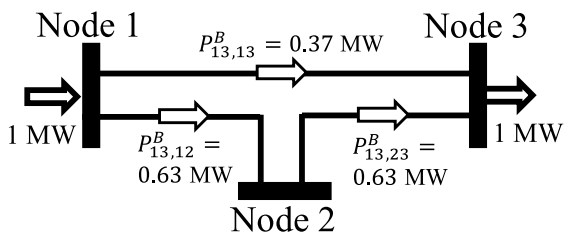

(c) Power transfer distribution factor example

Fig. 1: Illustrative test system for electrical distance estimations (normalized impedances)

than trading prices $\lambda_{n m}$. When generating perceived prices are lower than trading prices since parameters $\gamma_{n m}$ would be positive. The total money paid or received by agent $n$, for real power bilateral trades per time unit, is expressed by

$$
\Lambda_{n}=\sum_{m \in \omega_{n}} \lambda_{n m} p_{n m}-\underbrace{\left(\gamma_{n}^{0}+\sum_{m \in \omega_{n}} \gamma_{n m} p_{n m}\right)}_{=\Gamma_{n}}, n \in \Omega
$$

where $\Gamma_{n}$ is the part reserved to the system operator.

From the system operator's point of view, the total amount of money collected through network charges is simply given by $\Gamma_{\text {SO }}=\sum_{n \in \Omega} \Gamma_{n}$. As mentioned previously, note that the focus is put only on real power trades fees and not reactive power injection's. This money can be used to cover operation expenses - such as maintenance, power losses, power injection compensations, etc. - as well as investment cost when considering multiple time steps.

\section{B. Unique Cost Allocation Policy}

The way to allocate costs is to share them equally between community members. At the image of Paris' one-way trip public transportation ticket, no discrimination is made between trades. Because of the universality of this policy, agents in recurrently congested areas might not be spurred to behave in a responsible manner. Misbehavior of a few agents may penalize the rest of the community. If network charges are such that both ends of a trade are equally responsible, they can be written as

$$
\gamma_{n m}^{\text {uniq }}= \pm \frac{u^{\text {uniq }}}{2}, \quad \forall(n, m) \in \Omega \times \omega_{n},
$$

where the sign of $\gamma_{n m}^{\text {uniq }}$ is such that $\gamma_{n m}^{\text {uniq }} p_{n m} \geqslant 0$, so $\geqslant 0$ for producers and $\leqslant 0$ for consumers. Unique unit fee $u^{\text {uniq }}$ is expressed in $€ / M W h$ in the case of an hourly time unit.

\section{Electrical Distance Cost Allocation Policy}

To be more precise in how costs are allocated it is possible to use network charges proportional to the electrical distance between agents. As for cab travels, this cost allocation policy would incite agents to trade with their closest electrical partners. Such policy would reflect that long electric distances are costlier to operate due to power losses for example. However, power losses can not directly be considered as they are quadratic, which can not be superposed. When both ends of a trade equally share responsibility and the previous sign convention is followed, network charges become

$$
\gamma_{n m}^{\text {dist }}= \pm \frac{u^{\text {dist }} d_{n m}}{2}, \quad \forall(n, m) \in \Omega \times \omega_{n},
$$

where $\mathrm{d}_{n m}$ is the electrical distance between agent $n$ and agent $m$. Distance unit fee $u^{\text {dist }}$ is expressed in $€ / M W h /$ distance unit if the same time unit is used.

The definition of an electrical distance is a crucial issue for this cost allocation policy. [30] recommends two electrical distances, initially developed to allow a better vulnerability assessment through topological visualization of an electrical structure. It is possible to either consider

1) the Thevenin Impedance Distance, where each line is weighed by the norm of its Thevenin impedance after which a shortest path algorithm is performed to obtain the Thevenin electrical distance between two distant nodes, or

2) the Power Transfer Distance, where the absolute value of Power Transfer Distribution Factors induced by a unitary trade are summed.

Thevenin electrical distance $Z_{i l}^{\text {th }}$ between two connected nodes is evaluated by

$$
Z_{i l}^{\text {th }}=\left|Z_{i i}+Z_{l l}-Z_{i l}-Z_{l i}\right|
$$

where $Z_{i l}=1 / Y_{i l}$ is an element of bus and branch impedance matrix. If applied to the small example of Figure 1a, Thevenin impedances are as in Figure 1b. It is evident that multiple roads are possible for the power to flow between two nodes. But, e.g., for a power injection at node 1 and a withdrawal at node 3 only one Thevenin impedance distance value must prevail. It is proposed to use the shortest path as a metric to reflect the path on which the power exchange as the highest effect. For example, shortest path algorithm of [31] can be used. In the case of Figure 1b, Thevenin impedance distance between node 1 and node 3 is $d_{13}^{\text {th }}=\min \left(Z_{12}^{\text {th }}+Z_{23}^{\text {th }}, Z_{13}^{\text {th }}\right)=0.96$.

On the other hand, the power transfer distance between distant nodes is evaluated by

$$
d_{i l}^{\mathrm{PT}}=\sum_{(r, o) \in \mathcal{L}}\left|P_{i l, r o}^{B}\right|
$$

where $P_{i l, r o}^{B}$ is the power transfer distribution factor, in the sense of [32], of the branch from node $r$ to node $o$ for an injection at node $i$ and a withdrawal at node $l$. Power transfer distribution factors of Figure 1a for injection at node 1 and withdrawal at node 3 are given in Figure 1c. The resulting power transfer distance is $d_{13}^{\mathrm{PT}}=0.63+0.63+0.37=1.63$.

So the two approaches do not lead towards identical distance estimations. The Thevenin impedance distance, considering the shortest path, is more adapted to radial networks such as distribution grids. While the power transfer distance, considering a DC power flow approximation of the entire network, is better suited for meshed networks such as transmission grids. 
In consequence, the choice of electrical distance type has a strong impact on the efficiency of the electrical distance cost allocation policy.

\section{Uniform Zonal Cost Allocation Policy}

While the unique cost allocation policy does not differentiate agents, the electrical distance one might individualize too much grid tariffs. At the image of Danish public transportation system, using a zonal cost allocation policy seems a good compromise between both. In this situation the electrical network would be divided in several zones associated to distinct zonal unit fees. Each zone could be managed by a different system operator. A zone with a high price would incite outside agents not to trade with agents within, and push within agents towards self-consumption. In this sense, the zonal cost allocation policy allows to economically isolate an area. However, its efficiency strongly depends on zones' design.

A possible way to obtain the network charges is to sum zonal network fees of zones crossed by each trade. As mentioned previously, the electrical path is not unique which could lead to multiple lists of crossed zones. To select only one of them, the shortest electrical path criterion, as defined above with the Thevenin electrical distance, can be taken. then, chosen crossed zones would reflect the most stressed one by a trade. For illustrative simplicity, in this paper the mechanism is simplified by considering a uniform zonal unit fee. This way, the problem of how zonal unit fees are designed between zones is limited. When costs are equally shared on both ends of a trade and the sign convention is conserved, uniform zonal network charges become

$$
\gamma_{n m}^{\text {zone }}= \pm \frac{u^{\text {zone }} \mathrm{N}_{n m}^{\text {zone }}}{2}, \quad \forall(n, m) \in \Omega \times \omega_{n},
$$

where $\mathrm{N}_{n m}^{\text {zone }}$ corresponds to the number of crossed zone for trade $p_{n m}$. Zonal unit fee $u^{\text {zone }}$ is expressed in $€ / \mathrm{MWh}$.

Both electrical distance and zonal cost allocation policies depend on grid characteristics, supposed time independent. Their unit fees can be adapted to grid's status (e.g. between day and night). As any exogenous approach the proposed allocation policies may not ensure efficiency of the P2P market, as pointed in [16] in the case of transmission rights. Even though local marginal prices seem effective, they may be largely rejected for their opacity by $\mathrm{P} 2 \mathrm{P}$ market participants anxious for transparency. To define the unit fees, as well as zones, the system operator can periodically, e.g. yearly, update unit fees based on the revenue adequacy and the congestion occurrence rate of the last period. This type of historical data analysis is a common practice. For example the French transmission operator, RTE, publishes ${ }^{1}$ its transmission tariffs (or TURPE for Tarif d'Utilisation du Rseau Public d'Electricit) based on this type of analysis. More details on this method can be found in the "Study on tariff design for distribution systems" prepared for the European Commission. Alternatively, zones can follow administrative delimitation such as states. Note that

\footnotetext{
${ }^{1}$ https://clients.rte-france.com/lang/an/clients_producteurs/services_clients/ tarif.jsp

${ }^{2}$ https://ec.europa.eu/energy/sites/ener/files/documents/20150313\% 20Tariff\%20report\%20fina_revREF-E.PDF
}

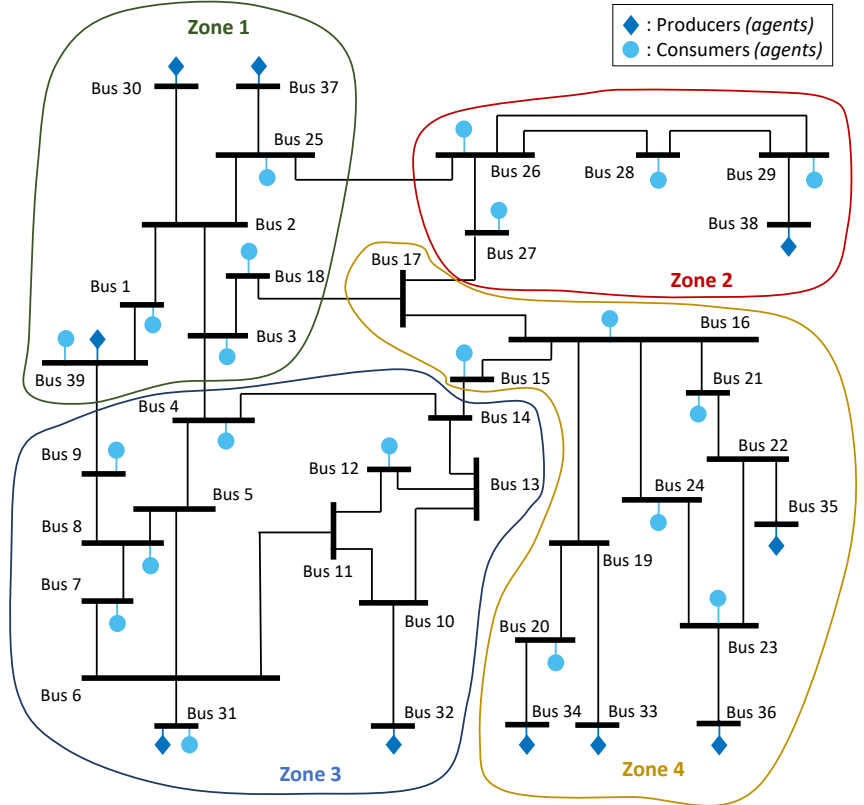

Fig. 2: New England test case for joint P2P market and OPF

other allocation policies have recently been proposed such as in [33].

\section{CAse-studies And Application Results}

This section evaluates the effects induced by the proposed cost allocation policies and compares them to a market free of network charges.

\section{A. Test Case}

To evaluate market responses flexible agents need to be defined. In addition, grid characteristics are needed to test feasibility of power commitments. Some test cases exist for P2P markets, such as in [9], but they do not provide power system's characteristics. Many standards exist for OPF problems on transmission and distribution networks. However, they do not involve flexible loads similar to flexible generation. Following the notion of organized prosumer group model, proposed in [3], the focus can be put on transmission systems such as the IEEE 39-bus test system.

Hence, there is a need for a novel test case adapted to study P2P markets accounting for network constraints. The IEEE 39bus test system is adapted to include flexible loads. Generators will keep their power boundaries. A wide flexibility range is given to consumers, with bounds from $10 \%$ to $150 \%$ of IEEE test system's fixed loads. The test case uses quadratic cost functions written as follows

$$
f_{n}\left(p_{n}\right)=\frac{1}{2} a_{n} p_{n}^{2}+b_{n} p_{n}
$$

Parameters $a_{n}$ and $b_{n}$, inspired from [9], are summarized in Table I. The final power system in Fig. 2 will be referred as the New England test case. The network is divided in four administrative zones close to the following states: Maine, Vermont, New Hampshire and Massachusetts. Not affecting market outcomes, constant terms $\gamma_{n}^{0}$ are set to zero. 
Cost allocation policies will, for example, exert differently on the trade between node 16's consumer and node 39's producer. Since this test case is based on a meshed network the power transfer distance is used. Note that in such case unit fee $u^{\text {dist }}$ is expressed in $€ / M W h$. The power transfer electrical distance between node 16 and 39 is 7.3 , without dimension. While Thevenin impedance distance's path, passing by nodes $\{16,17,18,3,2,1,39\}$, crosses two zones. This gives a ratio 7.3 between the electrical distance and uniform network charges, and a ratio of 2 in the case of zonal network charges.

\section{B. Free Market}

Free market refers to the P2P market without network charges. In the New England test case, the free market leads to an electricity price of $57.2 € / \mathrm{MWh}$ which is uniform as proven in Appendix A. Iterative process (6) converges in 9.5 seconds in MATLAB to primal and dual residuals below $10^{-4}$ when $\rho=1$. Independently from the power network, it is important to study interactions between participants. This aspect will allow to verify if the conjectures made, when cost allocation policies have been defined, are correct. Looking

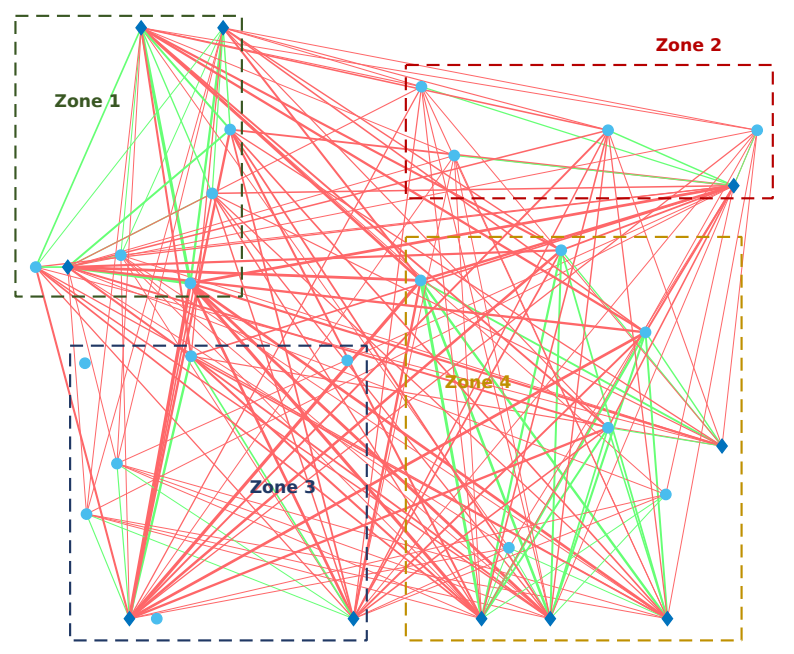

(a) Power trades free of network charges

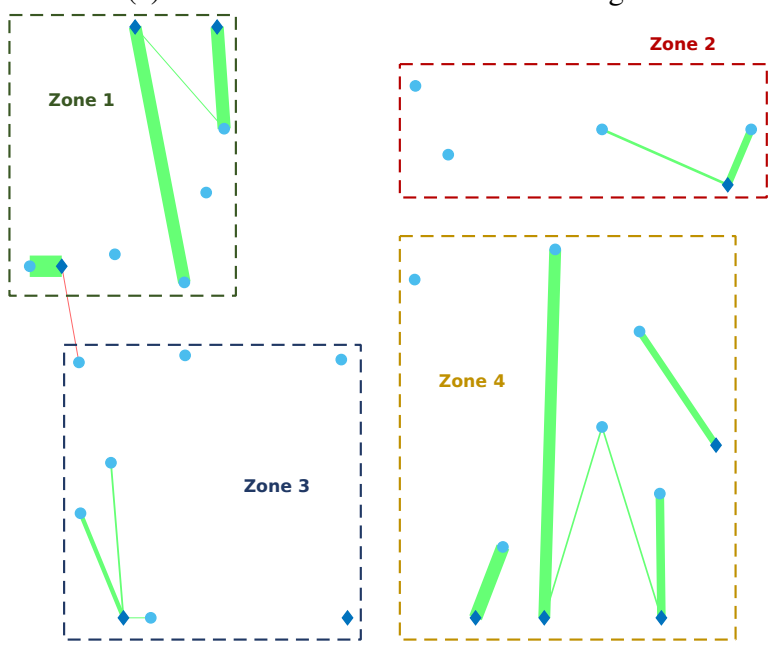

(c) Power trades for a distance unit fee $u^{\text {dist }}$ of $10 € / \mathrm{MWh}$ at how trades are distributed between participants and the exchanges between zones seem also relevant. Moreover, grid usage can be studied in a second step to point the presence of potential congestion.

To visualize the bilateral trades it is possible to look into graph theory visualizations such as interaction diagrams. However, their interpretation in the case of multiple bilateral trades might be complex. A more intuitive visualization is to show the trades on power system's map Fig. 2. For clarity reasons lines and buses will not be represented. To emphasize the difference between intra- and inter-zone exchanges, they will respectively be represented by green and red lines. To discriminate between main and small trades lines thickness will be proportional to the power traded. Finally, only trades over $10^{-2} \mathrm{MW}$ are represented in Fig. 3.

Fig. 3a shows free market power exchanges between participants. Agents almost equally trade with all their partners. This results in many inter-zone exchanges which implies no correlation between market and power system. In other words, agents do not favor local trades. As a consequence a high amount of power flows between zones. The global absolute exchanges between zones is above $2 \mathrm{GW}$. However, a DC

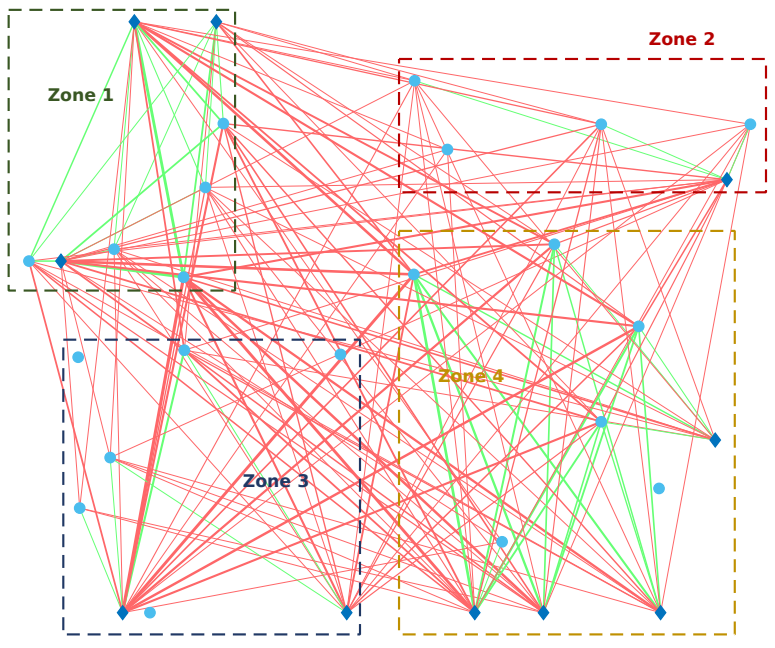

(b) Power trades for a unique unit fee $u^{\text {uniq }}$ of $10 € / \mathrm{MWh}$
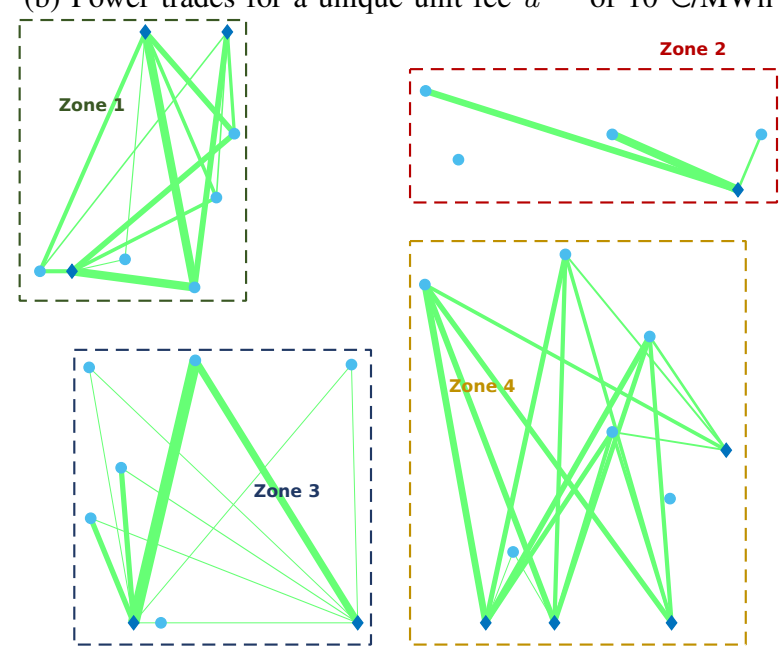

(d) Power trades for a uniform zonal unit fee $u^{\text {zone }}$ of $10 € / \mathrm{MWh}$

Fig. 3: Cost allocation policies' influence on trades (red lines: inter-zone exchanges, green lines: intra-zone exchanges) 


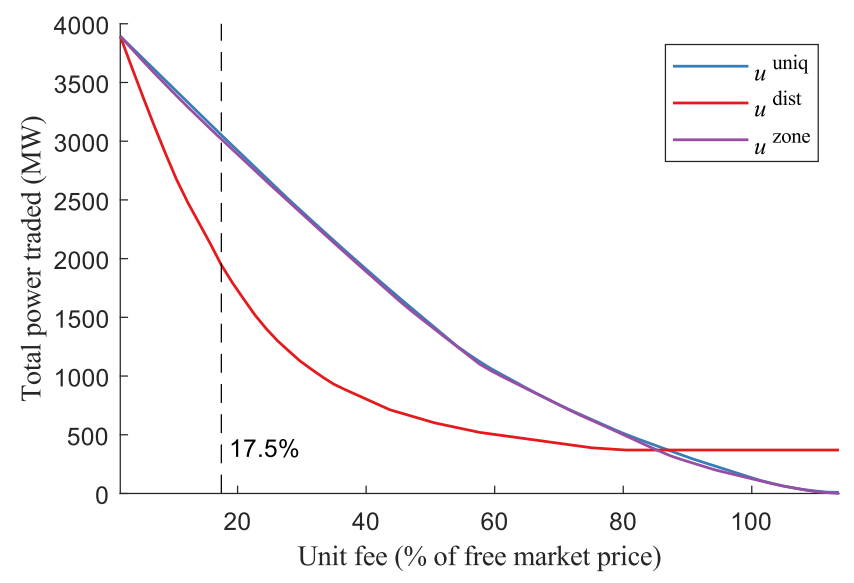

(a) Total amount of power traded on the market

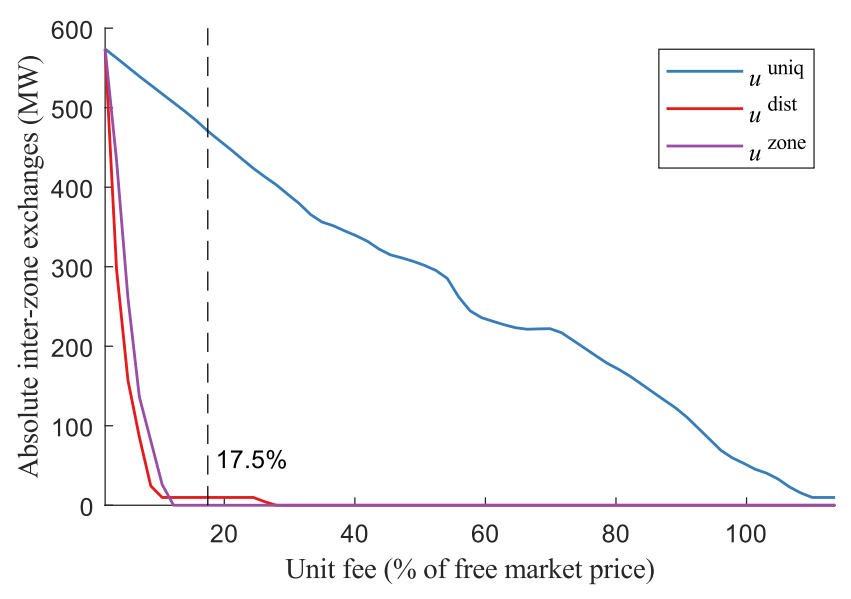

(b) Total amount of power exchanged between zones

Fig. 4: Sensitivities of power exchanges to unit fees $(17.5 \% \equiv 10 € / M W h)$

TABLE I: Agents' characteristics of New England test case

\begin{tabular}{|c|c|c|c|c|c|}
\hline Agent & Bus & $a_{n}\left[€ / \mathrm{MW}^{2}\right]$ & $b_{n}[€ / \mathrm{MW}]$ & $\underline{P_{n}}[\mathrm{MW}]$ & $\overline{P_{n}}[\mathrm{MW}]$ \\
\hline 1 & 1 & 0.067 & 64 & -146.4 & 0 \\
\hline 2 & 3 & 0.047 & 79 & -483 & 0 \\
\hline 3 & 4 & 0.047 & 71 & -750 & 0 \\
\hline 4 & 7 & 0.053 & 62 & -350.7 & 0 \\
\hline 5 & 8 & 0.082 & 65 & -783 & 0 \\
\hline 6 & 9 & 0.052 & 83 & $-9 . \overline{8}$ & 0 \\
\hline 7 & 12 & 0.087 & 63 & -12.8 & 0 \\
\hline 8 & 15 & 0.057 & 81 & -480 & 0 \\
\hline 9 & 16 & 0.050 & 73 & -493.5 & 0 \\
\hline 10 & 18 & 0.052 & 69 & -237 & 0 \\
\hline 11 & 20 & 0.071 & 62 & -1020 & 0 \\
\hline 12 & 21 & 0.064 & 79 & -411 & 0 \\
\hline 13 & 23 & 0.057 & 60 & -371.3 & 0 \\
\hline 14 & 24 & 0.082 & 80 & -462.9 & 0 \\
\hline 15 & 25 & 0.069 & 78 & -336 & 0 \\
\hline$\overline{16}$ & $-\overline{2}$ & 0.069 & $\overline{70}$ & -208.5 & $\overline{0}$ \\
\hline 17 & 27 & 0.086 & 62 & -421.5 & 0 \\
\hline 18 & 28 & 0.054 & 70 & -309 & 0 \\
\hline 19 & 29 & 0.078 & 66 & -425.3 & 0 \\
\hline 20 & 31 & 0.081 & 70 & -13.8 & 0 \\
\hline 21 & 39 & 0.059 & 71 & -1656 & 0 \\
\hline 22 & 30 & 0.089 & 18 & 0 & 1040 \\
\hline 23 & 31 & 0.067 & 21 & 0 & 646 \\
\hline 24 & 32 & 0.055 & 37 & 0 & 725 \\
\hline 25 & 33 & 0.082 & 25 & 0 & 652 \\
\hline$\overline{26}$ & $-\overline{-}$ & 0.088 & $\overline{17}$ & -- & 508 \\
\hline 27 & 35 & 0.076 & 38 & 0 & 687 \\
\hline 28 & 36 & 0.084 & 28 & 0 & 580 \\
\hline 29 & 37 & 0.077 & 36 & 0 & 564 \\
\hline 30 & 38 & 0.051 & 38 & 0 & 865 \\
\hline 31 & 39 & 0.087 & 19 & 0 & 1100 \\
\hline
\end{tabular}

power flow analysis shows only one congested line located between node 16 and node 19 , used up to $130 \%$ of its capacity. Hence the risk of congestion does not necessarily originate from exchanges between zones. Of course these quantitative, numerical results strongly depend on agents' characteristics.

\section{Impact on Peers' Interactions}

Free market results above manifest that no link exists between market operations and grid's infrastructure. Understand- ably, feasibility of market outcomes is not guaranteed. Even though commitments were applicable, the system operator would not be able to allocate operation costs without using network charges. The proposed cost allocation policies have a direct impact on relationships between community members and the increase of unit fees might incite agents to trade less or differently.

By definition the unique cost allocation policy does not discriminate trades. So, as shown in Fig. 3b, strong interactions between zones persist even with a unique unit fee of 10 $€ / \mathrm{MWh}$, which is equivalent to $17.5 \%$ of the free market electricity price. This policy does not encouraged agents to reach a dispatch coherent with the power system. However, the level of trades and the number of relevant trades sufferer from a small decrease. However the possibility to impact the grid power flow thank to a market fee is a general property.

On the other hand, with the electrical distance cost allocation policy, and the same value of unit fee, the number of relevant trades has plummeted, Fig. 3c. Strongest agents appear to privilege single partnerships with each other while others are left with many small, negligible trades, so below the threshold. This reflects that market participants are restrained to a lower level of flexibility than previously, analysis deepen in Section IV-D. Power interactions now appear to be grouped by area making it more coherent with the network. Note that this assertion is not strict as two inter-zone trades remain relevant.

Finally, with the zonal cost allocation policy similar interactions can be reached with the same value of unit fee, Fig. $3 \mathrm{~d}$. The pressure does not reach the same level of constraint on market's flexibility since more power is traded than in Fig. $3 \mathrm{c}$. In this case agents seem restrained to a small number of partnerships, instead of only one. Note that here no inter-zone trade continue to exist.

The sensitivities of power exchanges to unit fee variations, overall on the market and between zones, are presented in Fig. 4. Inspection of Fig. 4a reveals that the network charges actually decreases overall the traded volumes on the market which, as it will be detailed in Section IV-D, lowers the stress on the transmission network. Fig. 4a also shows that 


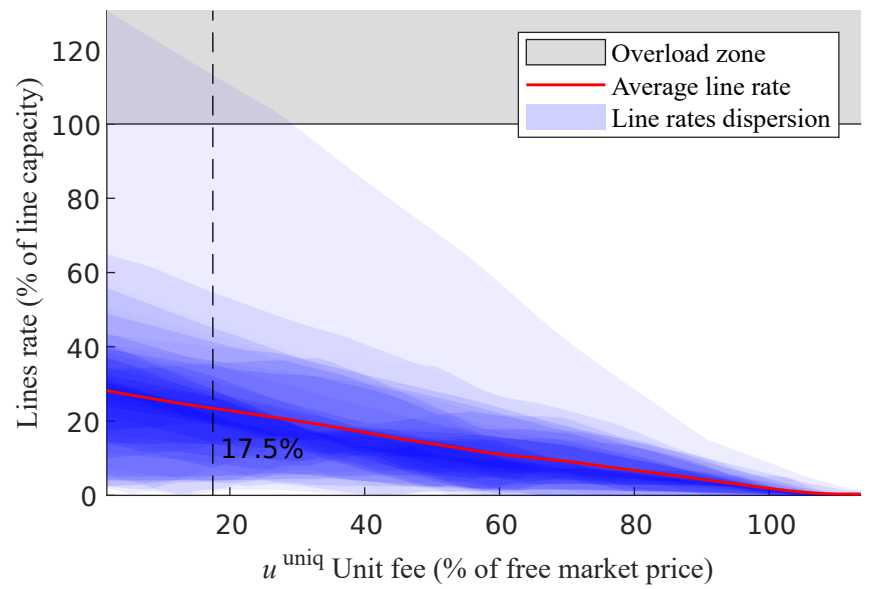

Fig. 5: Line rates of power trades with unique network charges $(17.5 \% \equiv 10 € / \mathrm{MWh})$

agents chose to opt-out of the market when unique and zonal network charges are too high. The electrical distance policy does not steer trades between partners connected to the same electrical node. Hence, agents 21 and 31, both connected to bus 39, continue to trade with each other even in presence of high distance unit fees. As expected, Fig. 4b shows that both electrical distance and zonal unit fees allow to annihilate all power exchanges between zones. This perfectly illustrate that, contrary to the unique cost allocation, electrical distance and zonal cost allocations enable to isolate zones economically.

\section{Effects on Power Network Usage}

Despite these results, market aggregation by zone does not reflect how the electrical grid is used. Cost allocation policies provides a tool for the system operator to affect the economic dispatch. Unit fees enable to tweak the expected outcomes of the P2P market in such way that line capacities might not be violated. They also offer to the system operator the chance to look for cost recovery.

Looking at power flows induced by power commitments is more relevant to evaluate the efficiency of cost allocation policies. The market does not follow any physical limitations unlike the electrical grid. Thus, the difference between a feasible and a none feasible market equilibrium lies in power network's feasibility set. In Fig. 5 DC power flows are represented for various unique unit fees by steps of $1 € / M W h$. For the given market community, in average, lines are used way below their capacity. As expected when the unit fee increases, lines usage decreases. However, one line risks congestion but a sufficient increase of unit fee allows its flow rate to fall below its capacity. More generally, the absolute disparity of line rates drops until market's flexibility is lost.

To compare the three cost allocation policies only the average (continuous) and the maximum (dash-dotted) line rates are plotted in Fig. 6 (upper part). It can easily be seen that electrical distance cost allocation policy behaves differently from the other two. As an indication, a zone corresponding to the possible range of the average line rate is shown. The lower

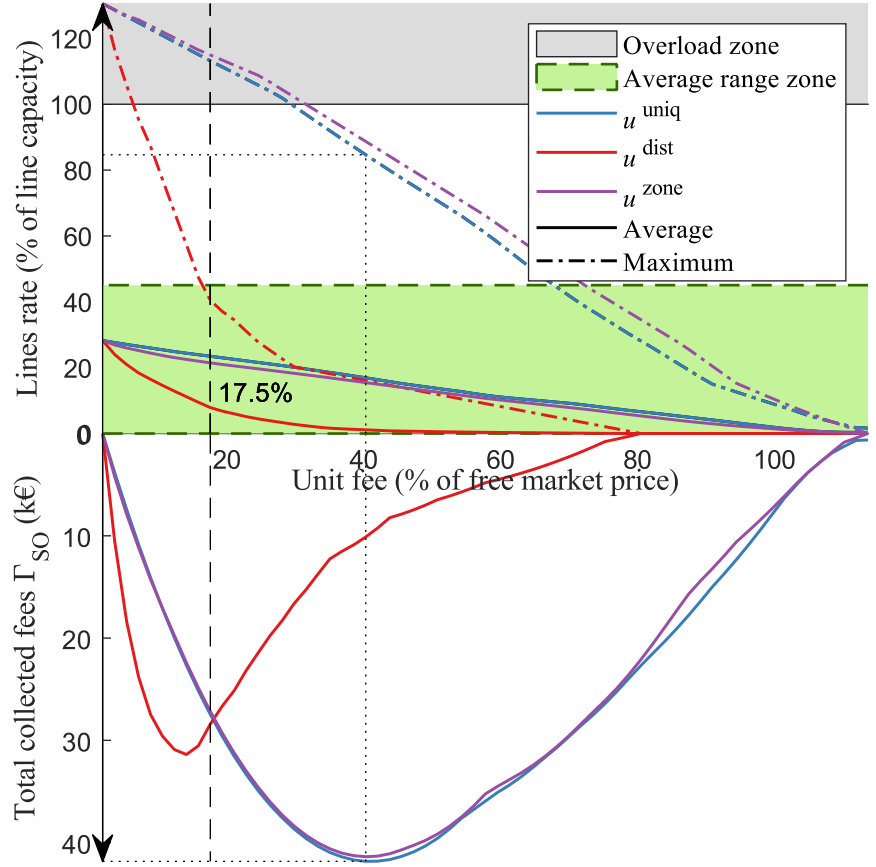

Fig. 6: Effects of unit fees on line rates (upper part) and total money collected by the system operator (lower part) $(17.5 \% \equiv 10 € / \mathrm{MWh})$

and upper bounds are respectively defined as the average line rate for the global minimum and maximum consumption.

Both maximum and average line rates decrease linearly with for unique and zonal network charges. Their behavior is linear as they are defined uniformly or uniformly over zones. As noted in Section IV-B the most stressed line is within a zone. In consequence, the zonal cost allocation policy is not able to remedy the congestion in a better way than the unique cost allocation policy as zones are not defined properly. The electrical distance cost allocation policy generates a greater impact on the market which is translated in a faster decrease not only on the average but also on the maximum line rate. This allows to obtain feasibility of market commitments with a lower over-cost for market participants. However, the money collected by the system operator might not be sufficient in this case to reach cost recovery.

The lower part of Fig. 6 represents the total amount of money collected by the system operator. From these curves it is clearly possible to identify when the market is too constrained, so when network charges are too high. In this configuration, agents choose to opt-out which leads to an absence of collected money. As a consequence it can be observed that a maximum exists, dashed lines for the unique cost allocation policy. Then, the system operator can deduce if operation costs can be recovered for a given cost allocation policy. Thus, Fig. 6 offers a graphic tool to the system operator. For a given cost allocation policy this helps choosing the unit fee to provide to market participants simply by following the proposed guide. For a given maximum acceptable line rate the corresponding unit fee and amount of money collected can be deduced. Or, for a given amount of money to collect the corresponding 


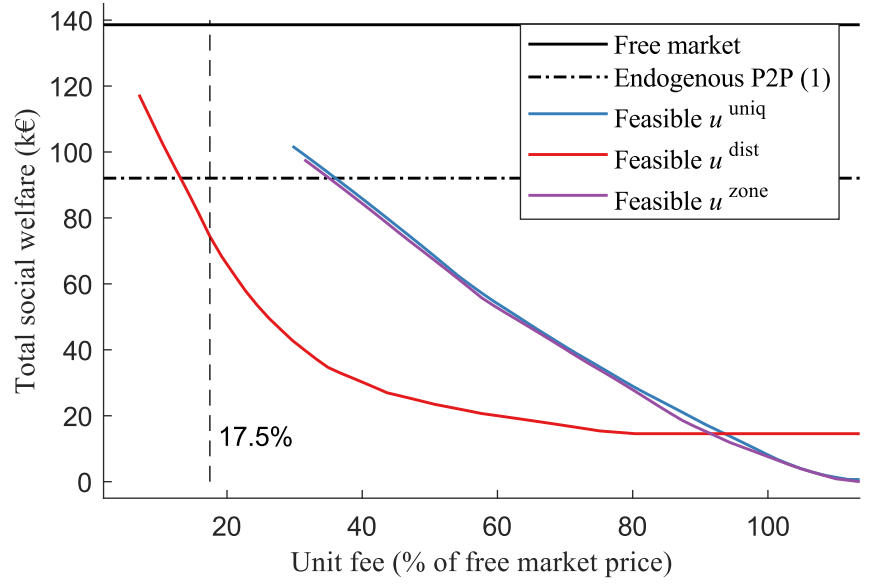

Fig. 7: Exogenous P2P market efficiency $(17.5 \% \equiv 10 € / \mathrm{MWh})$

unit fee and maximum line rate can be deduced. One can notice that distance-based network charges seems advisable when they are low compared to the operational cost while zonal, or even unique, network charges seems more suited for network charges larger than the operational cost. A more advance study would be required to determine whether this observation is related to the test case or intrinsic to network charges' design.

The proposed network charges affect bilateral trades in a way that may introduce sub-optimalities. As shown in Fig. 7 , considering network charges, when feasible, deteriorates clearing's optimality compared to the free market without network limitations. Distance-based network charges rapidly degrades the social welfare since low unit fees suffice to impact market outcomes, as observed in Fig. $4 \mathrm{~b}$ and 6. Remember that the proposed network charges encompass more than just congestion-related costs. Hence, exogenous P2P market (5) can neither be compared to the endogenous P2P economic dispatch (1) nor to [16]. If network charges were including only congestion-related costs, one with complete prior knowledge of the market could choose them optimally as in Appendix A. Since no distributed solution approach exists, (1) is handled with a centralized interior-point solver. In comparison to the classical economic dispatch, the proposed method based on network charges brings transparency and is simple to implement in P2P markets. However, this may be done at the cost of technical and economical drawbacks as respectively pointed in Fig. 6 and 7. This result reinforces the interest of developing a distributed approach solving (1) similarly to [5], which would require more involvement from the system operator.

\section{CONCLUSION}

Peer-to-peer markets are considered as a likely evolution of the power systems driven by distributed energy resources and ICT development. In this paper a peer-to-peer electricity market including network charges has been considered. Network charges, provided a priori, have been used as incentives to account for grid-related costs in a simple and transparent way. This mechanism incites market participants to respect power system's limits, rather than enforcing them. Tested for three incentive frameworks, on a novel test case based on the IEEE 39-bus test system, it has been shown the ability of this mechanism to limit the stress put on the physical grid by the market. Network charges also allow the system operator to collect money from market participants for their use of the grid in the aim of reaching cost recovery. On the down side, the approach may lead to inefficient or unfeasible solutions when network charges are not chosen wisely.

This exogenous approach is a candidate for a future implementation of peer-to-peer markets with low involvement of the system operator. In addition, the development of network charges adapted to distribution networks, so considering reactive powers, would provide a more generic exogenous peer-topeer market. As any consumer-centric system, it is essential to study the privacy and the security of market participants as well as the stability of the proposed design. In particular the presence of agents who could have the ability to be self sufficient represent a risk of snowballing effect. For each agent opting out, remaining agents would suffer from higher charges due to a redistribution between less participants. Finally, the resilience of the system to non-rational or strategic agents must be examined before a real world implementation.

\section{REFERENCES}

[1] C. Giotitsas, A. Pazaitis, and V. Kostakis, "A peer-to-peer approach to energy production," Technology in Society, vol. 42, pp. 28-38, 2015.

[2] D. Selloni, CoDesign for Public-Interest Services. Springer, 2017.

[3] Y. Parag and B. K. Sovacool, "Electricity market design for the prosumer era," Nature energy, vol. 1, p. 16032, 32016.

[4] F. F. Wu and P. Varaiya, "Coordinated multilateral trades for electric power networks: theory and implementation," International Journal of Electrical Power \& Energy Systems, vol. 21, no. 2, pp. 75-102, 1999.

[5] J. Qin, R. Rajagopal, and P. P. Varaiya, "Flexible Market for Smart Grid: Coordinated Trading of Contingent Contracts," IEEE Transactions on Control of Network Systems, vol. PP, no. 99, p. 1, 2017.

[6] G. Andersson, Modelling and Analysis of Electric Power Systems ETH Zurich, 2008.

[7] G. Hug and G. Andersson, "Decentralized Optimal Power Flow Control for Overlapping Areas in Power Systems," IEEE Transactions on Power Systems, vol. 24, no. 1, pp. 327-336, 22009.

[8] S. Kar, G. Hug, J. Mohammadi, and J. M. F. Moura, "Distributed State Estimation and Energy Management in Smart Grids: A Consensus $\{+\}$ Innovations Approach," IEEE Journal of Selected Topics in Signal Processing, vol. 8, no. 6, pp. 1022-1038, 122014.

[9] G. Hug, S. Kar, and C. Wu, "Consensus $\{+\}$ Innovations Approach for Distributed Multiagent Coordination in a Microgrid," IEEE Transactions on Smart Grid, vol. 6, no. 4, pp. 1893-1903, 72015.

[10] T. Erseghe, "Distributed Optimal Power Flow Using ADMM," IEEE Transactions on Power Systems, vol. 29, no. 5, pp. 2370-2380, 92014.

[11] S. Boyd, N. Parikh, E. Chu, and J. Eckstein, "Distributed optimization and statistical learning via the Alternating Direction Method of Multipliers," Foundations and Trends in Machine Learning, vol. 3, no. 1, pp. $1-122,2010$.

[12] J. Liu, M. Benosman, and A. U. Raghunathan, "Consensus-based distributed optimal power flow algorithm," in 2015 IEEE Power \& Energy Society Innovative Smart Grid Technologies Conference (ISGT). IEEE, 2 2015, pp. 1-5.

[13] T. Liu, X. Tan, B. Sun, Y. Wu, X. Guan, and D. H. K. Tsang, "Energy management of cooperative microgrids with $\mathrm{P} 2 \mathrm{P}$ energy sharing in distribution networks," in 2015 IEEE International Conference on Smart Grid Communications (SmartGridComm). IEEE, 11 2015, pp. 410415.

[14] A. Kargarian, J. Mohammadi, J. Guo, S. Chakrabarti, M. Barati, G. Hug, S. Kar, and R. Baldick, "Toward Distributed/Decentralized DC Optimal Power Flow Implementation in Future Electric Power Systems," IEEE Transactions on Smart Grid, vol. PP, no. 99, p. 1, 2017. 
[15] E. Munsing, J. Mather, and S. Moura, "Blockchains for decentralized optimization of energy resources in microgrid networks," in 2017 IEEE Conference on Control Technology and Applications (CCTA), 8 2017, pp. 2164-2171.

[16] F. Wu, P. Varaiya, P. Spiller, and S. Oren, "Folk theorems on transmission access: Proofs and counterexamples," Journal of Regulatory Economics, vol. 10, no. 1, pp. 5-23, 71996.

[17] R. H. Day, "Rational choice and economic behavior," Theory and Decision, vol. 1, no. 3, pp. 229-251, 31971.

[18] E. Sorin, L. A. Bobo, and P. Pinson, "Consensus-based approach to peer-to-peer electricity markets with product differentiation," submitted to IEEE Transactions on Power Systems, 2017.

[19] L. Liu and Z. Han, "Multi-Block ADMM for Big Data Optimization in Smart Grid," CoRR, 2015.

[20] W. Deng, M.-J. Lai, Z. Peng, and W. Yin, "Parallel Multi-Block ADMM with O(1/k) Convergence,” J. Sci. Comput., vol. 71, no. 2, pp. 712-736, 52017.

[21] M. Hong, Z.-Q. Luo, and M. Razaviyayn, "Convergence Analysis of Alternating Direction Method of Multipliers for a Family of Nonconvex Problems," SIAM Journal on Optimization, 2014.

[22] M. Hong and Z.-Q. Luo, "On the linear convergence of the alternating direction method of multipliers," Mathematical Programming, vol. 162, no. 1, pp. 165-199, 32017.

[23] H. Wang, A. Banerjee, and Z.-Q. Luo, "Parallel Direction Method of Multipliers," in Advances in Neural Information Processing Systems 27, Z. Ghahramani, M. Welling, C. Cortes, N. D. Lawrence, and K. Q. Weinberger, Eds. Curran Associates, Inc., 2014, pp. 181-189.

[24] A. Tarantola, Inverse Problem Theory and Methods for Model Parameter Estimation. Society for Industrial and Applied Mathematics, 12005.

[25] F. Siddiqui, S. Zeadally, C. Alcaraz, and S. Galvao, "Smart Gric Privacy: Issues and Solutions," in 2012 21st International Conference on Computer Communications and Networks (ICCCN). IEEE, 7 2012, pp. 1-5.

[26] P. McDaniel and S. McLaughlin, "Security and Privacy Challenges in the Smart Grid," IEEE Security \& Privacy Magazine, vol. 7, no. 3, pp. 75-77, 52009.

[27] J. Liu, Y. Xiao, S. Li, W. Liang, and C. L. P. Chen, "Cyber Security and Privacy Issues in Smart Grids," IEEE Communications Surveys \& Tutorials, vol. 14, no. 4, pp. 981-997, 242012.

[28] E. Farhi and I. Werning, "Monetary Policy, Bounded Rationality, and Incomplete Markets," National Bureau of Economic Research, Tech. Rep. 23281, 32017.

[29] H. Yang, M. Zhang, and M. Lai, "Complex dynamics of Cournot game with bounded rationality in an oligopolistic electricity market," Optimization and Engineering, vol. 12, no. 4, pp. 559-582, 122011.

[30] P. Cuffe and A. Keane, "Visualizing the Electrical Structure of Power Systems," IEEE Systems Journal, vol. 11, no. 3, pp. 1810-1821, 92017.

[31] E. W. Dijkstra, "A Note on Two Problems in Connexion with Graphs," Numerische Mathematik, vol. 1, no. 1, pp. 269-271, 1959.

[32] R. D. Christie, B. F. Wollenberg, and I. Wangensteen, "Transmission management in the deregulated environment," Proceedings of the IEEE vol. 88, no. 2, pp. 170-195, 22000.

[33] J. Zhao, J. Lu, and K. Lo, "A Transmission Congestion Cost Allocation Method in Bilateral Trading Electricity Market," Energy and Power Engineering, vol. 9, no. 4B, pp. 240-249, 2017.

\section{APPENDIX}

\section{A. Comparison With The Pool-based Market And The Endoge-} nous Economic Dispatch

The Lagrangian of the real power market part of (5)

$$
L_{\rho}(\mathbf{P}, \mathbf{W}, \boldsymbol{\Lambda}, \boldsymbol{\mu})=\sum_{n \in \Omega} L_{n, \rho}\left(P_{n}, \mathbf{W}, \Lambda_{n}, \mu_{n}\right)
$$

with $\mathbf{W}=\left(w_{n m}\right)_{n, m}, \boldsymbol{\Lambda}=\left(\lambda_{n m}\right)_{n, m}$ and $\boldsymbol{\mu}=\left(\mu_{n}\right)_{n \in \Omega}$. Local augmented objectives can be written as

$$
\begin{array}{r}
L_{n, \rho}\left(P_{n}, \mathbf{W}, \Lambda_{n}, \mu_{n}\right)=\tilde{f}_{n}\left(p_{n}\right)+\mu_{n}\left(\sum_{m \in \omega_{n}} p_{n m}-p_{n}\right) \\
+\gamma_{n}^{0}+\sum_{m \in \omega_{n}}\left[\gamma_{n m} p_{n m}+\lambda_{n m}\left(\left(w_{n m}-w_{m n}\right) / 2-p_{n m}\right)\right. \\
\left.+(\rho / 2)\left(\left(w_{n m}-w_{m n}\right) / 2-p_{n m}\right)^{2}\right] \quad(17)
\end{array}
$$

where $\rho>0$ is the penalty factor, $P_{n}=\left(p_{n m}\right)_{m \in \omega_{n}}$ and $\Lambda_{n}=\left(\lambda_{n m}\right)_{m \in \omega_{n}}$. Function $\tilde{f}_{n}$ is the extended-value of $f_{n}$, in the sense of [11], with a domain defined by (5c)-(5g). Karush-Kuhn-Tucker (KKT) stationarity conditions of (16)(17) allows to obtain equalities

$$
\mu_{n}=\left\{\begin{array}{l}
\frac{\partial \tilde{f}_{n}}{\partial p_{n}} \\
\lambda_{n m}-\gamma_{n m} \quad m \in \omega_{n}
\end{array}, n \in \Omega\right.
$$

Note that the perceived price $\mu_{n}$, which is the dual variable of constraint (5c), links agent $n$ 's energy cost $\frac{\partial \tilde{f}_{n}}{\partial p_{n}}$ to trading prices $\left(\lambda_{n m}\right)_{m \in \omega_{n}}$ and network charges $\left(\gamma_{n m}\right)_{m \in \omega_{n}}$.

KKT optimality conditions of (1) can be written

$$
\mu_{n}=\left\{\begin{array}{l}
\frac{\partial \tilde{f}_{n}}{\partial p_{n}} \\
\lambda_{n m}-\eta_{i \in \Omega^{n}} \quad m \in \omega_{n}
\end{array}, n \in \Omega\right.
$$

where $\eta_{i \in \Omega^{n}}$ denotes the dual variable of nodal balance constraint (1i) associated to the node $i$ on which agent $n$ is connected. If one had complete prior knowledge of the market, they could solve the endogenous P2P economic dispatch (1) and deduce the optimal nodal energy prices, namely $\gamma_{n m}=\eta_{i \in \Omega^{n}}$. However, in doing so they would only be able to recover their costs of congestion but neither taxes nor other operation costs as proposed in this paper.

In a pool market, consensus constraint $(5 b)$ is replaced by power balance constraint $\sum_{n \in \Omega} p_{n}=0$ and constraints (5c)$(5 \mathrm{~g})$ are non-existent. Hence, KKT stationarity conditions of the pool market read

$$
\frac{\partial \tilde{f}_{n}}{\partial p_{n}}+\lambda^{\mathrm{PM}}=0
$$

where dual variable $\lambda^{\mathrm{PM}}$ of the power balance constraint represents the pool market energy price. As both (5) and the pool market would be made of the same agents, one could readily notice that (5) without network charges, so with $\left(\gamma_{n m}\right)_{n, m}=0$, leads to a uniform trading price equal to the pool market price $\lambda^{\mathrm{PM}}$.

\section{B. Decentralized P2P Market ADMM}

ADMM of (5) consists in iterations

$$
\begin{aligned}
\{\mathbf{P}, \boldsymbol{\mu}\}^{k+1} & =\underset{\mathbf{P}, \boldsymbol{\mu}}{\operatorname{argmin}} L_{\rho}\left(\mathbf{P}, \mathbf{W}^{k}, \boldsymbol{\Lambda}^{k}, \boldsymbol{\mu}\right) \\
\mathbf{W}^{k+1} & =\underset{\mathbf{W}}{\operatorname{argmin}} L_{\rho}\left(\mathbf{P}^{k+1}, \mathbf{W}, \boldsymbol{\Lambda}^{k}\right) \\
\boldsymbol{\Lambda}^{k+1} & =\boldsymbol{\Lambda}^{k}+\rho\left(\left(\mathbf{W}^{k+1}-\mathbf{W}^{k+1, \mathbf{T}}\right) / 2-\mathbf{P}^{k+1}\right)
\end{aligned}
$$

Looking at the Lagrangian formulation (16)-(17), step (21a) can be distributed among agents such as

$$
\left\{P_{n}, \mu_{n}\right\}^{k+1}=\underset{P_{n}, \mu_{n}}{\operatorname{argmin}} L_{n, \rho}\left(P_{n}, \mathbf{W}^{K}, \Lambda_{n}^{k}, \mu_{n}\right)
$$

As in [11] update (21b) can be written

$$
\mathbf{W}^{k+1}=\left(\mathbf{P}^{k+1}-\mathbf{P}^{k+1, \boldsymbol{T}}\right) / 2-\left(\boldsymbol{\Lambda}^{k+1}-\boldsymbol{\Lambda}^{k+1, \boldsymbol{T}}\right) /(2 \rho)
$$

Substituting (23) in (21c) gives $\boldsymbol{\Lambda}^{k+1}-\boldsymbol{\Lambda}^{k+1, \boldsymbol{T}}=0$. So, after the first iteration $\mathbf{W}^{k+1}=\left(\mathbf{P}^{k+1}-\mathbf{P}^{k+1, \boldsymbol{T}}\right) / 2$. Overall, after simplifications, the decentralized negotiation mechanism reads as (6). 